\title{
Structure Asymmetry Effects in the Optical Gain of Piezostrained InGaN Quantum Wells
}

\author{
L.-H. Peng, Member, IEEE, Y.-C. Hsu, and C.-W. Chuang
}

\begin{abstract}
We investigate the effects of structural asymmetry on the electronic and optical properties of indium gallium nitride (InGaN) quantum wells (QW's). Using a pulsed current excitation technique, spectral blue shift as large as $80 \mathrm{meV}$ is observed in a strained 3.0-nm $\operatorname{In}_{0.2} \mathrm{Ga}_{0.8} \mathrm{~N}$ QW as the pulsed current increases from $1 \mathrm{~mA}$ to $1 \mathrm{~A}$. Based on a self-consistent calculation, we are able to quantify a gain competition process among the interactions of piezoelectricity, many-body, charge screening, and band filling effects. Such interactions are shown to provide a mechanism for shaping the QW confined potential such that superior carrier confinement and charge screening of the piezoelectric field can be obtained in the asymmetric InGaN QW. At high carrier injection of $N_{\mathrm{inj}}>2 \times 10^{19} \mathbf{c m}^{-3}$, a tenfold increase in the TE-polarized optical gain can be achieved by using the asymmetric GaN-InGaN-AIGaN QW instead of the symmetric InGaN-AlGaN QW. Due to the diminishing of piezoelectricityinduced quantum-confined Stark effect, the calculated optical gain spectra of the asymmetric InGaN QW exhibit a spectral blue shift with respect to those of the symmetric InGaN QW.
\end{abstract}

Index Terms - InGaN quantum well, optical gain, piezoelectric effects, structural asymmetry.

\section{INTRODUCTION}

$\mathbf{R}$ ECENT progress [1] in the epitaxial growth, doping control, and device process of the wide-bandgap III-V nitrides have realized this material system a promising light emitter spanning the spectral range from ultraviolet [2] to amber [3]. Success of Nichia's high-brightness blue/green indium gallium nitride ( $\mathrm{InGaN})$ quantum well $(\mathrm{QW})$ light-emitting and violet laser diodes represent one such exciting achievement [4]; while the more recent reports on high-power and longlifetime continuous-wave $(\mathrm{CW})$ room-temperature operation of InGaN laser diodes on sapphire [5] and gallium nitride $(\mathrm{GaN})[6]$ substrates have stimulated further investigation on the device designing issues.

Despite InGaN QW is one of the most applicable materials used in the active layer design of laser diodes, the corresponding emission mechanisms are still not fully understood yet. In particular, many of its novel properties such as manybody [7] and piezoelectric [8] effects still remain a great challenge to be resolved. As a first-order investigation of the quantum-confined strained InGaN structures, conventional

Manuscript received December 11, 1998; revised April 8, 1999. This work was supported by the National Science Council under Grant 88-2212-M-002032 and Grant 89-2215-E-002-023.

L.-H. Peng is with the Department of Electrical Engineering and Institute of Electro-Optical Engineering, National Taiwan University, Taipei, Taiwan, R.O.C.

Y.-C. Hsu and C.-W. Chuang are with the Institute of Electro-Optical Engineering, National Taiwan University, Taipei, Taiwan, R.O.C.

Publisher Item Identifier S 1077-260X(99)05694-4. wisdom has pursued a model analysis based on a wurtzitetype Rashba-Sheka-Pikus (RSP) Hamiltonian in calculating the valence band structures [9]. In this method, one typically neglects the materials intrinsic properties such as many-body and piezoelectric effects in order to give a guideline on the device designing issues. In doing so, the corresponding electronic band structures and optical properties can be evaluated in the so-called flat-band (FB) free-carrier model [10]. Albeit simple in the mathematical formulation, one has found great discrepancy between the measurements and the calculations. For example, an emission peak energy difference on the order of $200 \mathrm{meV}$ has been noted in a InGaN QW blue emitter when compared with the bandgap of a stress-free bulk epilayer [11]. Plausible causes of such a bandgap narrowing has been ascribed to the exciton and strain effects but no further theoretical analysis has been confirmed [12].

In order to explain the phenomenon of bandgap narrowing, several groups have attributed the spontaneous emission from InGaN QW's grown on buffered sapphire substrates to the recombination of exciton localized at certain potential minima. The lateral size of which can vary from less than $60 \mathrm{~nm}$ in a quantum disk-like shape to $300 \mathrm{~nm}$ in a segmentedQW fashion due to the In compositional inhomogeneity [13]. Assuming the gain region containing the potential minima or localized states are sufficiently large such that the quantum confinement remains essentially in the epitaxial direction, Chow et al. have shown that the effects due to the bandgap inhomogeneity formed by nonrandom alloy compositional fluctuation can be approximated by a statistical average of a normal distribution [14].

Recent investigations on the emission mechanism of bulk $\mathrm{GaN}$ and InGaN QW's prepared by lateral epitaxial overgrowth (LEO), however, have revealed the cause of carrier localization is not due to the In phase separation initiated by the threading dislocations, instead it is due to the effective bandgap fluctuation formed by point defects or growth parameters [15]. On the other hand, other groups have pointed out the piezoelectric field in the strained InGaN QW plays an important role in determining the energy position and the oscillator strength of the optical transitions [16]. By analyzing the time-resolved photoluminescence (TRPL) spectra of the intra- and interwells emissions in InGaN QW's, Kollmer et al. have concluded the explanation based on localized states is not feasible [17]. Instead, the observations of very long delayed time and multiple low energy emission can be consistently explained by considering the effect of piezoelectric field in strained InGaN QW's. 
Piezoelectricity occurs in a wurtzite-type QW due to a strain-induced polarization in the longitudinal (growth) direction, e.g., $c$-axis [18]. Piezoelectric field of this kind is known to point from the growth surface toward the substrate and is on the order of $1 \mathrm{MV} / \mathrm{cm}$ for a low In composition InGaN-GaN QW [19]. Piezoelectricity also plays an important role in tailoring the material's optical and electronic properties. For example, a novel piezoelectricity-induced quantum-confined Stark effect (PQCSE) has recently come to attention [20]. In addition to reduce the QW oscillator strength, PQCSE also results in a spectral red shift [21] and enhancement of decay time in the PL spectra [22]. Schottky barrier height enhancement [23] and high electron mobility transistors [24] are, among others, the nitride-based devices that have taken advantage of such piezoelectric effects.

To date most of the PQCSE investigation on III-V nitrides have been limited to the case of few carrier injection such as studied by the PL measurements [19]-[22]. They do not encompass the important category upon which the lasing of InGaN QW takes place at high levels of carrier injection. As a result, study of the piezoelectric effects on the electron-hole plasma recombination process has become an important issue in resolving the lasing mechanism of InGaN QW lasers [25].

The interaction of electron-hole plasma in semiconductors is known as the many-body effects [26]. Many-body effects due to the attractive Coulomb interaction can give rise to the excitonic enhancement of interband transition probability [17], whereas those due to the repulsive Coulomb interaction are known to introduce the effects of bandgap renormalization [27], and charge screening. However, there are restrictions regarding the carrier injection density, and electric field strength beyond which the excitons can no longer survive at room temperature [13]. For a bulk GaN, such a critical charge density $N_{\text {crit }}$ is estimated to be about $1 \times 10^{18} \mathrm{~cm}^{-3}$ according to Debye-Huckel screening [7], [13]. In a highly excited QW, the excitonic phase becomes unstable as the two-dimensional (2-D) electron-hole density exceeds $n_{c}=1 / \pi a_{B}^{2}$, where $a_{B}$ is the exciton Bohr radius [28], [29].

On the other hand, the critical field strength needed to dissociate the free exciton in a GaN QW has been estimated to be about $6 \times 10^{5} \mathrm{~V} / \mathrm{cm}$ [30]. We note such a high field is naturally associated with the growth of piezostrained InGaN heterostructures. For example, from the Franz-Keldysh oscillations in the photoreflectance (PR) measurements, Wetzel et al. are able to determine a piezoelectric field of $0.63 \mathrm{MV} / \mathrm{cm}$ in a $\operatorname{In}_{0.18} \mathrm{Ga}_{0.82} \mathrm{~N}-\mathrm{GaN}$ heterostructure [16]. The cause of spectral red-shifting of the PL spectra with respect to the PR optical bandgap has therefore been attributed to transitions due to the field-induced tilt band edges in InGaN.

With the inherent piezoelectricity, one expects the effect of PQCSE to dominate the band structures, and the recombination and transport properties of strained nitride structures. Here, we present a use of pulsed current excitation technique to study the piezoelectric and many-body effects on the optical properties of InGaN-GaN QW's. A series of electroluminescence (EL) experiments on strained $\mathrm{GaN}-\mathrm{In}_{0.2} \mathrm{Ga}_{0.8} \mathrm{~N}-\mathrm{Al}_{0.2} \mathrm{Ga}_{0.8} \mathrm{~N}$ QW and on $\mathrm{Zn}: \mathrm{Si}_{\mathrm{In}} \mathrm{In}_{0.06} \mathrm{Ga}_{0.94} \mathrm{~N}$ double-heterostructure (DH) emitters in the high carrier injection regime are reported. A spectral blue shift as large as $80 \mathrm{meV}$ in the QW emitter is observed as the current injection increases from $1 \mathrm{~mA}$ to 1 A.

By analyzing the gain competition process due to the effects of PQCSE, bandgap renormalization, band filling, and charge screening in a self-consistent scheme, we are able to quantify the phenomenon of carrier-induced spectral blue shifting in a piezostrained InGaN QW for the first time. In the low injection regime of $N_{\mathrm{inj}}<5 \times 10^{18} \mathrm{~cm}^{-3}$, the In compositional inhomogeneity has found little influence on the InGaN QW emission spectrum. At high carrier injection, however, the inhomogeneous spectral broadening of the InGaN QW emission is found correlated with the In compositional inhomogeneity.

It is also found the interaction of the above perturbations can provide a mechanism for shaping the quantum confined potential. Superior carrier confinement, and charge screening of PQCSE can be realized in an asymmetric GaN-InGaN-AlGaN QW compared with the symmetric AlGaN-InGaN-AlGaN QW. As a result, it is shown the asymmetric InGaN QW can provide a peak gain coefficient that is one order of magnitude higher than that of the corresponding symmetric InGaN QW.

\section{EXPERIMENT}

Samples used in this study were grown by metalorganic chemical vapor deposition (MOCVD) on the c-face of sapphire substrates; the active region of the light-emitting diode (LED) structure consisted of an undoped 3.0-nm strained $\mathrm{In}_{0.2} \mathrm{Ga}_{0.8} \mathrm{~N}$ QW sandwiched between a n-type $\mathrm{Si}: \mathrm{GaN}$ and a p-type $\mathrm{Mg}: \mathrm{Al}_{0.2} \mathrm{Ga}_{0.8} \mathrm{~N}$ layer of a thickness of $4 \mu \mathrm{m}$ and $100 \mathrm{~nm}$, respectively. The detailed growth procedure and characteristic emission spectra of the QW emitter at forward current less than $100 \mathrm{~mA}$ have been described in [31]. In order to investigate the many-body effects on the InGaN QW emission properties, a pulsed current excitation technique was used in the measurement. In addition, another reference set of experiments were performed on $\mathrm{Si}: \mathrm{Zn}: \mathrm{In}_{0.06} \mathrm{Ga}_{0.94} \mathrm{~N}-\mathrm{Al}_{0.15} \mathrm{Ga}_{0.85} \mathrm{~N}$ double-heterostructure (DH) emitters [32] such that the effects of piezoelectricity and dimensionality on the bandgap renormalization can be carefully examined.

The devices were biased with pulsed currents up to $1.5 \mathrm{~A}$ at a pulsewidth of $0.5 \mu \mathrm{s}$ and a low repetition rate of $10 \mathrm{~Hz}$. With a duty cycle as low as $10^{-\tilde{5}}$, one thereby was able to eliminate the heating effects and avoid any artifacts in the spectrum analysis. For optical characterization of the InGaN emitters, a grating spectrometer equipped with a calibrated Si photodiode and a boxcar analysis technique were used in the measurements [33]. All of the above experiments were conducted at room temperature.

\section{RESUlT AND DisCUSSION}

\section{A. Optical Characterization}

We illustrate in Fig. 1 the EL spectra of the strained 3.0-nm $\mathrm{In}_{0.2} \mathrm{Ga}_{0.8} \mathrm{~N} \mathrm{QW}$ at various levels of pulsed current injection. While the emission intensity of the 3.0-nm InGaN QW in Fig. 1(a) increases with the current injection, a spectral blue 


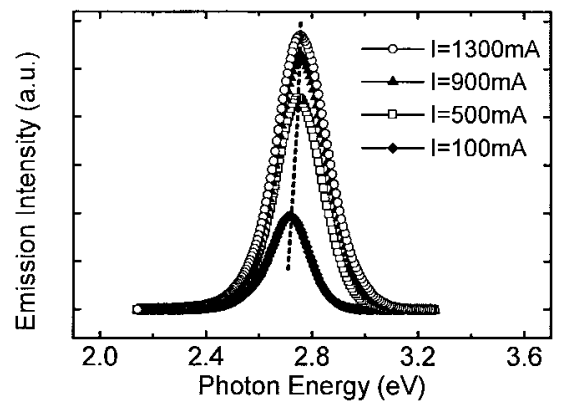

(a)

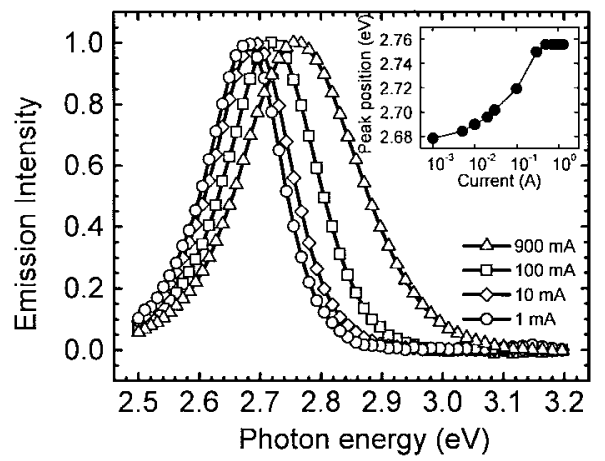

(b)

Fig. 1. (a) Emission spectra and (b) normalized EL spectra of a 3.0-nm $\mathrm{In}_{0.2} \mathrm{Ga}_{0.8} \mathrm{~N} \mathrm{QW}$ emitter at various injection currents.

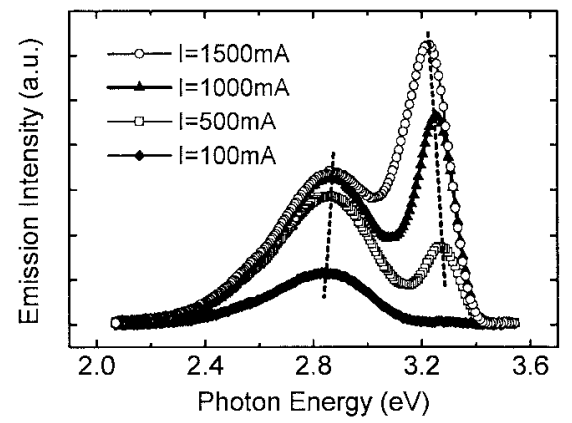

Fig. 2. Emission spectra of a $\operatorname{In}_{0.06} \mathrm{Ga}_{0.94} \mathrm{~N} \mathrm{DH}$ emitter at various injection currents.

shift as large as $80 \mathrm{meV}$ is clearly resolved in Fig. 1(b) as the injected current increases from $1 \mathrm{~mA}$ to $1 \mathrm{~A}$. In comparison, those from the band edge emission at $3.3 \mathrm{eV}$ and broad blue emission at $2.8 \mathrm{eV}$ from the 50-nm Si:Zn: $\operatorname{In}_{0.06} \mathrm{Ga}_{0.94} \mathrm{~N}$ DH structure shown in Fig. 2 appear to have opposite spectral shifting. The spectral blue shifting of the broad blue emission is known to result from the band filling effects on the $\mathrm{Zn}$ related deep levels [34]. Its emission intensity, however, saturates at an injected current level of $1 \mathrm{~A}$ and suggests a limited $\mathrm{Zn}$-impurity concentration in InGaN DH emitters [35]. On the other hand, the increase of the $3.3 \mathrm{eV}$ band edge emission intensity and the corresponding red shifting of the emission spectra with injection current suggest a dominant band-to-band optical transition process [36], [37]. We note the spectral red shifting in the band edge emission represents a well-known phenomenon of many-body bandgap renormalization in bulk InGaN [38].
Combined with the information shown above, two important issues characterizing the emission properties of InGaN QW still remain unsolved. The first is the so-called bandgap narrowing indicating a spectral red shift of the QW emission peak energy with respect to the bandgap of bulk InGaN. The other is the spectral blue shifting of the InGaN QW emission with current injection. The latter resembles a gain competition process between the bandgap renormalization and band filling effects that are known to conventional III-V QW lasers [39]. Due to the fact that bandgap renormalization scales with the reduced dimensionality [40], the spectral blue shifting observed in the piezostrained InGaN QW emission represents an interesting fundamental phenomenon to be resolved.

\section{B. Theoretical Modeling}

Since the phenomena of bandgap narrowing and spectral blue shifting are pertinent to the operation of $\mathrm{InGaN} \mathrm{QW}$ emitter, it is desirable to resolve these properties from a fundamental point of view. For analyzing the electronic and optical properties of strained InGaN QW's, it is desirable to develop a computational scheme that is capable of treating the effects of In compositional inhomogeneity, PQCSE, many-body bandgap renormalization, band filling, and charge screening on an equal footing. Shown in Appendix are the equations that have been used to calculate the band structures and optical properties of the InGaN QW's studied in this work.

In the self-consistent calculation, the equation of motion for the conduction band Schrodinger equation and valence band RSP Hamiltonian are solved by an iteration coupled to the Poisson equation whose spatially varying piezoelectric field is concurrently screened by the charge distribution in the QW region. Regarding the many-body bandgap renormalization on nitride-based QW's, an important universal relation between the reduced bandgap and carrier density, i.e., $\Delta E_{g} \propto n_{2 D}^{1 / 3}$ has recently been reported by Park and Chuang [27]. They have shown that this relation is independent of the QW thickness, and the material properties. Evaluation of the InGaN QW bandgap renormalization can thus be taken by using this universal relation curve.

In analyzing the effects of In compositional inhomogeneity, we approximate the nonrandom alloy fluctuation by a statistical average of a normal distribution in the QW plane [14]. In particular, the effect of monolayer fluctuation in the $\mathrm{InGaN}$ QW thickness is neglected such that one can focus on the In inhomogeneity effects. In modeling the piezoelectric field, we follow the notation of [41] shown in (A4) such that a correct polarity of the polarization field is ensured. For the case of piezostrained InGaN-GaN heterostructure, the direction of the piezoelectric field is to point from the InGaN epilayer toward the $\mathrm{GaN}$ substrate [16].

In the calculation, the material parameters of $\mathrm{InGaN}$ and $\mathrm{AlGaN}$ are taken as a linear interpolation from those of the binary GaN [42], AlN [43], and InN [44] as listed in Table I, except the deformation potentials of AlN and $\mathrm{InN}$ are assumed to be the same as those of GaN. The equations of motion for electrons and holes are then solved by using a finite-difference method similar to that discussed in [45]. 


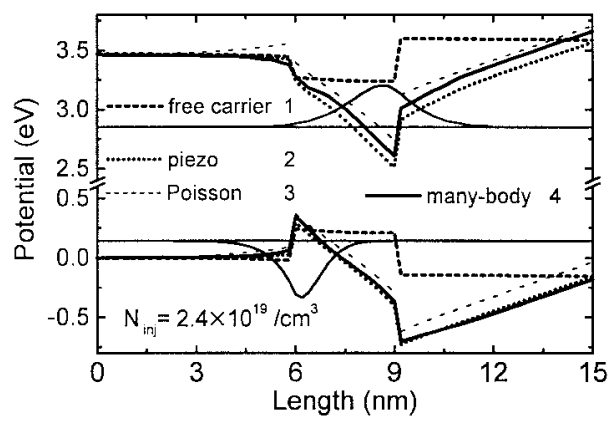

Fig. 3. Evolution of the $\mathrm{GaN}-\mathrm{In}_{0.2} \mathrm{Ga}_{0.8} \mathrm{~N}-\mathrm{Al}_{0.2} \mathrm{Ga}_{0.8} \mathrm{~N}$ QW confined potential as the perturbation is sequentially turned on and added up in the order of 1) free-carrier, 2) piezoelectricity, 3) charge screening (Poisson effect), and 4) many-body bandgap renormalization.

\section{Band Structure Analysis}

We depict in Fig. 3 the potential profile of the 3.0-nm strained $\mathrm{GaN}-\mathrm{In}_{0.2} \mathrm{Ga}_{0.8} \mathrm{~N}-\mathrm{Al}_{0.2} \mathrm{Ga}_{0.8} \mathrm{~N} \mathrm{QW}$ and compare it with the free-carrier FB model calculation. In the latter analysis, a square confined potential has been used. In the selfconsistent calculation, the confined potential profile evolves according to the perturbations incorporated in the analysis. In deriving Fig. 3, the perturbations are sequentially turned on and added to the Hamiltonian of (A1)-(A4) in the order of: 1) free carrier; 2) piezoelectricity; 3) charge screening; and 4) many-body bandgap renormalization. The calculation is made with an injected carrier density $N_{\mathrm{inj}}=2.4 \times 10^{19} \mathrm{~cm}^{-3}$ in the InGaN well region.

Data shown in Fig. 3 reveal how critically the QW potential profile evolves with the perturbations and indicate the importance of incorporating a self-consistent analysis. We also illustrate in Fig. 3 the $k_{\|}=0$ envelope function of the $n=1$ conduction $C_{1}$ and heavy-hole $H H_{1}$ subbands. It is noted the conduction subbands are more susceptible to the interaction with PQCSE and charge screening such that a quantum shift as large as $0.4 \mathrm{eV}$ occurs with respect to the unperturbed band edge. This observation agrees well with a recent tight-binding analysis on the charge screening of the piezoelectric field in a symmetric InGaN-GaN QW [46]. Such a mechanism will be shown later to be responsible for bandgap narrowing that was previously unsolved in the FB model analysis [11], [12].

\section{Optical Analysis}

1) In Compositional Inhomogeneity: The issues of In compositional inhomogeneity have been vividly discussed in the literature. It has been suggested the presence of large potential fluctuation causes a Stokes red-shift known as the band edge energy difference between the absorption and emission spectrum of InGaN QW's [13]. In order to examine this hypothesis, we calculate the inhomogeneous spontaneous emission spectra of the 3.0-nm $\operatorname{In}_{0.2} \mathrm{Ga}_{0.8} \mathrm{~N}$ QW according to (A7) and (A10). Here, a mean In value of 0.2 is assumed and a statistical average of a normal distribution with a standard deviation up to $\sigma=0.02$ is resumed. We also vary the carrier injection density from low $\left(N_{\mathrm{inj}}=1 \times 10^{18} \mathrm{~cm}^{-3}\right)$ to high $\left(N_{\mathrm{inj}}=4 \times 10^{19}\right.$ $\mathrm{cm}^{-3}$ ) in order to examine the In inhomogeneity effect on the linewidth broadening issues. In the calculation, a constant
TABLE I

Material Parameters For GaN, AIN, AND InN

\begin{tabular}{llll}
\hline Parameters & GaN & AIN & InN \\
\hline $\begin{array}{llll}\text { Lattice constant }(\AA) \\
\quad\end{array}$ & 3.189 & 3.112 & 3.544 \\
Energy parameters & & & \\
$\quad \mathrm{E}_{8}(\mathrm{cV})$ at $300^{\circ} \mathrm{K}$ & 3.44 & 6.28 & 1.95 \\
$\quad \Delta_{1}(\mathrm{meV})$ & 16 & -58.5 & 17 \\
$\quad \Delta_{\mathrm{to}}=3 \Delta_{2}=3 \Delta_{3}(\mathrm{meV})$ & 12 & 20.4 & 3 \\
$\begin{array}{l}\text { Conduction-band effective masses } \\
m_{e}^{*} / m_{0}\end{array}$ & 0.20 & 0.33 & 0.11 \\
$m_{e}^{t} / m_{0}$ & 0.18 & 0.25 & 0.11
\end{tabular}

Valence-band effective mass parameters $A_{1}$ $\begin{array}{cccc}A_{2} & -0.91 & -0.27 & -0.60 \\ A_{3} & 5.65 & 3.68 & 8.68\end{array}$ $\begin{array}{llll}A_{3} & 5.65 & 3.68 & 8.68\end{array}$ $\begin{array}{llll}A_{4} & -2.83 & -1.84 & -4.34 \\ A_{5} & -3.13 & -1.95 & -4.32\end{array}$ $\begin{array}{llll}A_{6} & -4.86 & -2.91 & -6.08\end{array}$

Deformation potentials (cV) $a_{c} \quad-4.08$ $\begin{array}{ll}D_{1} & 0.7 \\ D_{2} & 2.1\end{array}$

$\begin{array}{ll}D_{3} & 1.4 \\ D_{4} & -0.7\end{array}$

Elastic Stiffness constants (GPa)

$C_{11}$

$C_{12}$

$C_{13}$

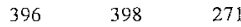

4

Dielectric constants $\begin{array}{lll}0.0 & 8.5 & 15.3\end{array}$

Piezoelectric constant $(\mathrm{cm} / \mathrm{V})$ $d_{31}$ $-1.7 \times 10^{-10}-2.0 \times 10^{10}-1.1 \times 10^{-10}$

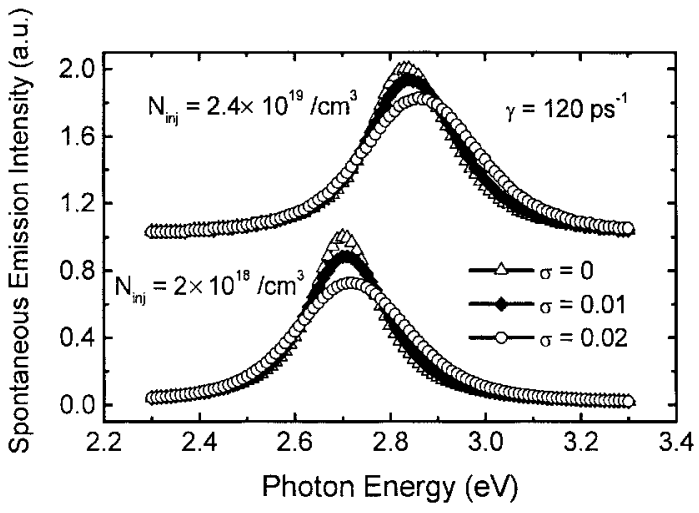

Fig. 4. Calculated spontaneous emission spectra of a 3.0-nm $\operatorname{In}_{0.2} \mathrm{Ga}_{0.8} \mathrm{~N}$ QW injected at $N_{\text {inj }}=2 \times 10^{18}$ and $2.4 \times 10^{19} \mathrm{~cm}^{-3}$. The In compositional inhomogeneity varies up to $\sigma=0.02$.

spectral line width broadening factor $\gamma=120 \mathrm{ps}^{-1}$ [47] is used in (A8) and In compositional variation within $\pm 6 \sigma$ of the mean value is incorporated in the band structure and optical gain analysis. We also neglect the monolayer thickness fluctuation such that one can focus on the effects of bandgap inhomogeneity on the emission properties.

Shown in Fig. 4 are the calculated spontaneous emission spectra of the 3.0-nm $\operatorname{In}_{0.2} \mathrm{Ga}_{0.8} \mathrm{~N}$ QW injected at $N_{\mathrm{inj}}$ of $2.4 \times 10^{19}$ and $2 \times 10^{18} \mathrm{~cm}^{-3}$, respectively. We first note a $130 \mathrm{meV}$ spectral blue shift is observed at $\sigma=0$ as the carrier injection density is increased by an order of magnitude. Considering a high In compositional variation of $\sigma=0.02$, a spectral blue shift on the order of $20 \mathrm{meV}$ with respect to the 


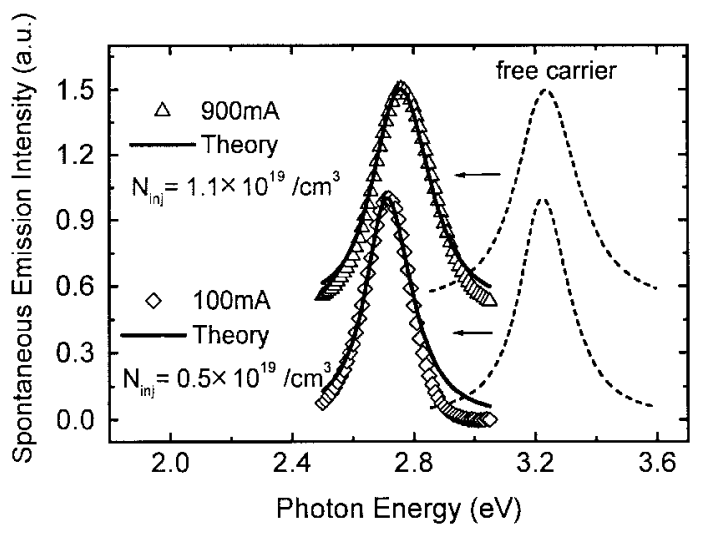

Fig. 5. Comparison between the calculated emission spectra and the experimental data of $900 \mathrm{~mA}$, and $100 \mathrm{~mA}$ from Fig. 1(b).

homogeneously broadened spectrum of $\sigma=0$ is resolved. We attribute this phenomenon to the fact that PQCSE dramatically decreases the optical gain of high In-content QW [15], [16]. As the statistical average is taken over a normal distribution of In composition, only those within $3 \sigma$ of the mean Incontent and on the low In composition side make significant contribution to the emission spectrum. Since the low In-content InGaN QW has optical gain spectrum on the higher energy side, such an average results in a spectral blue shift of 20 $\mathrm{meV}$ with respect to the homogeneously broadened spectrum at $\sigma=0$. In inhomogeneity therefore cannot be used to explain the bandgap narrowing effect which typically measures a spectral red shift of $200 \mathrm{meV}$ [11], nor can it be used to explain the large spectral blue shift $\sim 100 \mathrm{meV}$ with the increase of current injection [33]. We note previous work by Chow et al. [14] has neglected the effect of PQCSE in the analysis such that the averaged optical gain is dominated by the contribution from high In-content QW and revealed a spectral red shift.

On the other hand, our calculation indicates the inhomogeneous spectral broadening observed at high current injection is associated with the In compositional inhomogeneity. This is due to the real space filling of various In-content segmentedQW's as the injection current increases [13]. We note at $\sigma=0$, only homogeneous broadening due to the band-filling in the momentum space contributes to the spectral line width. At $N_{\text {inj }}=2.4 \times 10^{19} \mathrm{~cm}^{-3}$, for example, we find the full width at half maximum (FWHM) of the calculated spontaneous emission spectrum in Fig. 4 increases from $210 \mathrm{meV}$ at $\sigma=0$ to $290 \mathrm{meV}$ if $\sigma=0.02$ is resumed.

2) El Spectrum Analysis: We depict in Fig. 5 the calculated spontaneous emission spectra of the 3.0-nm InGaN QW blue emitter and compare the results with the experimental data in Fig. 1(b) under a pulsed current excitation at 900 and $100 \mathrm{~mA}$, respectively. In the self-consistent analysis, only the injected carrier density of $N_{\mathrm{inj}}=1.1 \times 10^{19} \mathrm{~cm}^{-3}$ and $5 \times 10^{18} \mathrm{~cm}^{-3}$, and a constant linewidth broadening factor $\left(\gamma=120 \mathrm{ps}^{-1}\right)$ [47] are chosen as the fitting parameters. The inhomogeneous spectral broadening in the high carrier injection regime is resumed with an In inhomogeneity of $\sigma=0.01$.

By doing so, we have found excellent agreement with the experimental data over a wide spectral range of 0.5

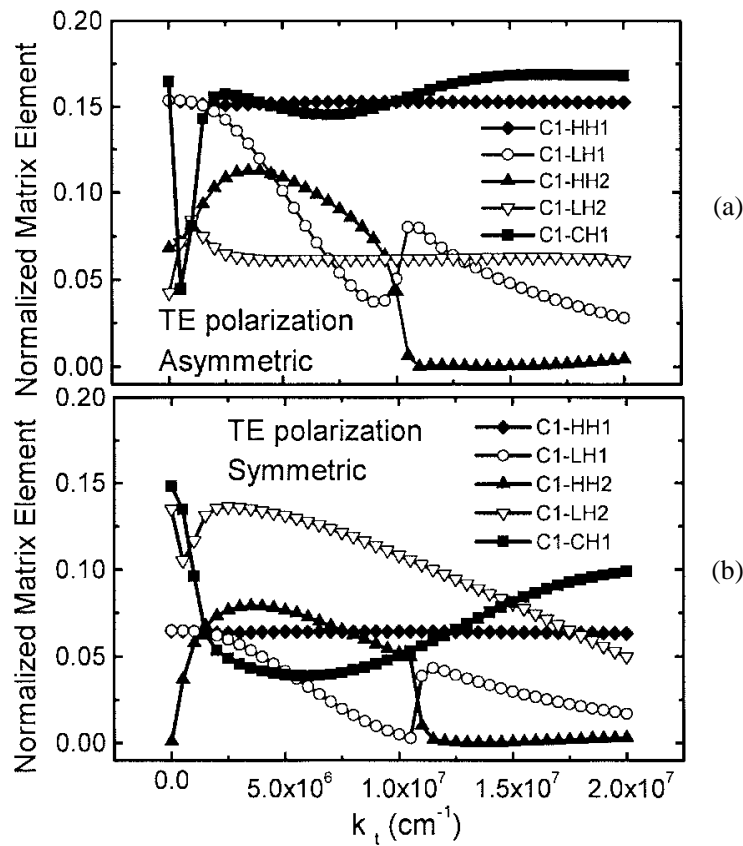

Fig. 6. Normalized momentum matrix elements in the TE-polarization for the 3.0-nm (a) asymmetric and (b) symmetric $\operatorname{In}_{0.2} \mathrm{Ga}_{0.8} \mathrm{~N}$ QW at $N_{\text {inj }}=4 \times 10^{19} \mathrm{~cm}^{-3}$.

$\mathrm{eV}$. One thereby can rationalize the causes of the so-called bandgap narrowing previously unsolved in the free-carrier FB model analysis [11] is due to the neglect of PQCSE, charge screening, band-filling, and bandgap renormalization in the simplified model calculation. In the self-consistent analysis, it is recognized the EL spectral blue shift with driving current is due to a dominant contribution from the charge screening and diminishing of the PQCSE effect.

\section{E. Structural Asymmetry Effects}

From the above analysis, it is shown the effects of PQCSE and many-body bandgap renormalization play an opposite role in red-shifting the emission spectrum of InGaN QW against those blue-shifting due to the charge screening and band filling effects. A good control of these mechanisms in the active layer design can thus lead to InGaN QW laser operation with desirable gain profile. In the following, we will discuss the use of structural symmetry effects to achieve such a goal. For the asymmetric GaN-InGaN-AlGaN QW, the InGaN active layer and $\mathrm{AlGaN}$ barrier are subject to compressive- and tensile-strain, respectively. On the other hand, for the symmetric InGaN-AlGaN QW, only the active layer of $\mathrm{InGaN}$ is strained such that the calculation can be compared with previous investigations [27]-[29]. In the self-consist analysis, we have previously shown it suffices to incorporate the first five valence subbands of heavy hole $\left(H H_{1}\right.$, and $\left.H H_{2}\right)$, light hole $\left(L H_{1}\right.$, and $\left.L H_{2}\right)$, crystal field splitting hole $\left(\mathrm{CH}_{1}\right)$, and the first two conduction subbands $\left(C_{1}\right.$, and $\left.C_{2}\right)$ in calculating the transition matrix elements and optical gain spectra [33].

1) Symmetry Effects on TE-Polarized Transition: Shown in Fig. 6 are the normalized TE-polarized momentum matrix elements calculated for InGaN QW's of both kinds of 


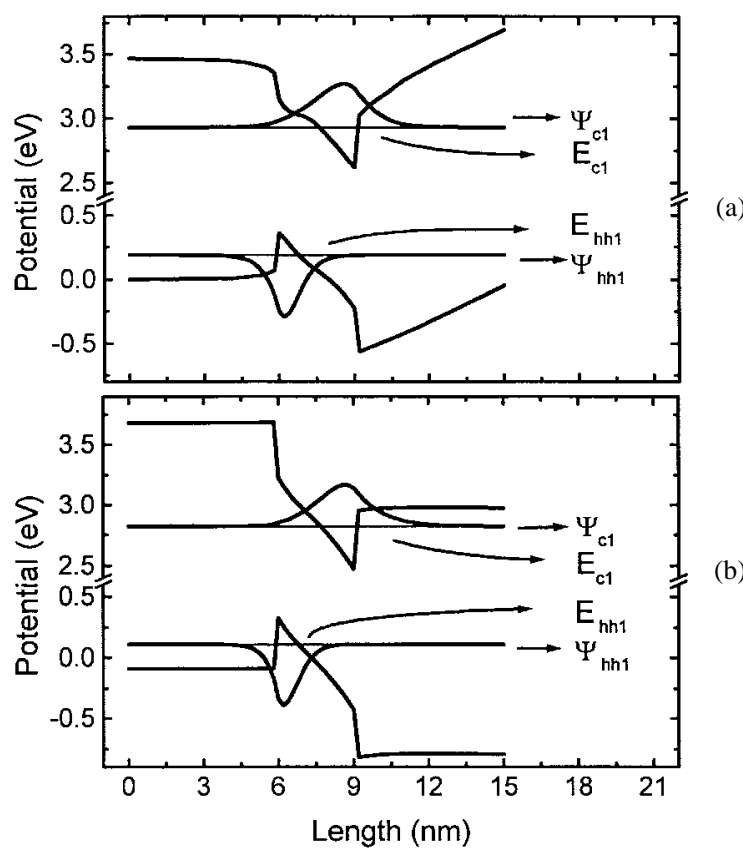

Fig. 7. Confined potential and subband envelope function of the 3.0-nm (a) asymmetric and (b) symmetric $\operatorname{In}_{0.2} \mathrm{Ga}_{0.8} \mathrm{~N}$ QW at $N_{\mathrm{inj}}=4 \times 10^{19} \mathrm{~cm}^{-3}$

symmetry. In the calculation, the following parameters of $N_{\text {inj }}=4 \times 10^{19} \mathrm{~cm}^{-3}$, and an undoped $\mathrm{In}_{0.2} \mathrm{Ga}_{0.8} \mathrm{~N}$ active layer of thickness $3 \mathrm{~nm}$, and undoped $\mathrm{Al}_{0.2} \mathrm{Ga}_{0.8} \mathrm{~N}$ barrier of $6 \mathrm{~nm}$, respectively, are used. We first note the design of an asymmetric InGaN QW can support a dominant TE-polarized $C_{1}-H H_{1}$ and $C_{1}-L H_{1}$ matrix elements whose magnitude are superior to those of the symmetric QW by a factor of 2.5. From (A6), it is known the QW momentum matrix element is proportional to the spatial overlapping between the subband envelope functions. The dramatic increase of such quantity from one to another indicates the structural symmetry, together with the interaction of carrier- and straininduced perturbations, can substantially alter the quantum confinement potential of the InGaN QW.

In supporting this analysis, we illustrate in Fig. 7 the corresponding confined potential and the envelope function of $C_{1}$ and $H H_{1}$ subband at both kinds of QW symmetry. We first note the interaction due to the carrier- and strainedinduced perturbations is such that the asymmetric InGaN QW can provide a better carrier confinement than does the symmetric QW. For example, the "center of mass" of the $C_{1}$ envelope function in the symmetric InGaN QW of Fig. 7(b) has a coordinate position of $8.512 \mathrm{~nm}$ and $H H_{1}$ of $6.292 \mathrm{~nm}$, respectively. In comparison, the corresponding value of $C_{1}$ in the asymmetric QW is $8.234 \mathrm{~nm}$ which is much closer to that of $H H_{1}$ at $6.266 \mathrm{~nm}$ in Fig. 7(a). This leads to a stronger subband overlapping in the latter such that a larger $C_{1}-H H_{1}$ matrix element can be achieved in the asymmetric InGaN QW.

We note that the effect of PQCSE is to reduce the strength of momentum matrix element and hence the oscillator strength [22]. Were PQCSE not taken into account, the normalized $\mathrm{C}_{1}-\mathrm{HH}_{1}$ matrix element would resume a theoretical value of 1.5 [28]. We further note that charge screening can provide an

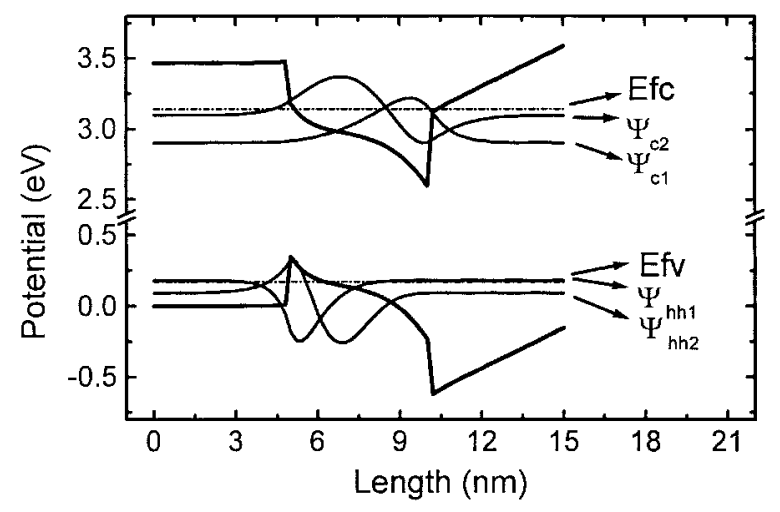

Fig. 8. Confined potential and subband envelope function of a 5.0-nm asymmetric $\mathrm{In}_{0.2} \mathrm{Ga}_{0.8} \mathrm{~N} \mathrm{QW}$ at $N_{\mathrm{inj}}=4 \times 10^{19} \mathrm{~cm}^{-3}$.

efficient process in reducing the piezoelectric effect [17] if a suitable structural symmetry is added to the active layer design. Shown in Fig. 8 are the calculated confinement potential and envelope function up to $n=2$ subband for a 5 -nm asymmetric InGaN QW at $N_{\mathrm{inj}}=4 \times 10^{19} \mathrm{~cm}^{-3}$. The interaction between the carrier-confinement, bandgap renormalization, band-filling, charge screening, and PQCSE is shown to result in a smoothing of the piezoelectric field in a wider InGaN QW. For a wide InGaN QW width under high carrier injection, it is expected the diminishing of PQCSE and increasing of subband overlapping will support a stronger momentum matrix element.

2) Effects of Structural Symmetry on Optical Gain: One important issue regarding the operation of strained InGaN QW laser is the designing of the active layer. It not only plays an important role in shaping the optical gain, but also affects the threshold current density. Here we consider the symmetry effects on the optical gain spectra of $\operatorname{In}_{0.2} \mathrm{Ga}_{0.8} \mathrm{~N}$ QW's. In order to examine the structural symmetry effects, In inhomogeneity is neglected in the following discussion.

Shown in Fig. 9(a) are the well width dependence of the TEpolarized peak gain coefficient calculated for the asymmetric and symmetric QW injected at $N_{\mathrm{inj}}=2 \times$ and $4 \times 10^{19}$ $\mathrm{cm}^{-3}$, respectively. Using an asymmetric instead of symmetric InGaN QW design, we note one order of magnitude increase in the optical gain coefficient can be achieved. This is ascribed to the larger TE-polarized matrix elements shown in Fig. 6 and smaller quasi-Fermi energy $E_{f v}$ separation to the valence subband in the asymmetric InGaN QW. For example, in a 3.0-nm asymmetric InGaN QW injected at $N_{\mathrm{inj}}=4 \times 10^{19}$ $\mathrm{cm}^{-3}$, we obtain a smaller value of $E_{f v}-E_{H H 1}=3.5$ $\mathrm{meV}$ compared with the much larger value of $45 \mathrm{meV}$ in the symmetric QW.

At $N_{\mathrm{inj}}=2 \times 10^{19} \mathrm{~cm}^{-3}$, we note the InGaN QW gain coefficient increases as the well width decreases. This is caused by the fading of PQCSE in the narrow InGaN wells of both symmetry. On the other hand, at a higher carrier injection density of $N_{\mathrm{inj}}=4 \times 10^{19} \mathrm{~cm}^{-3}$, a concave well width dependence of the peak gain coefficient occurs. This is due to a gain competition process such that band filling and screening of piezoelectric field take a leading term and result in a spectral blue shifting and enhancement of the QW optical gain at wider 


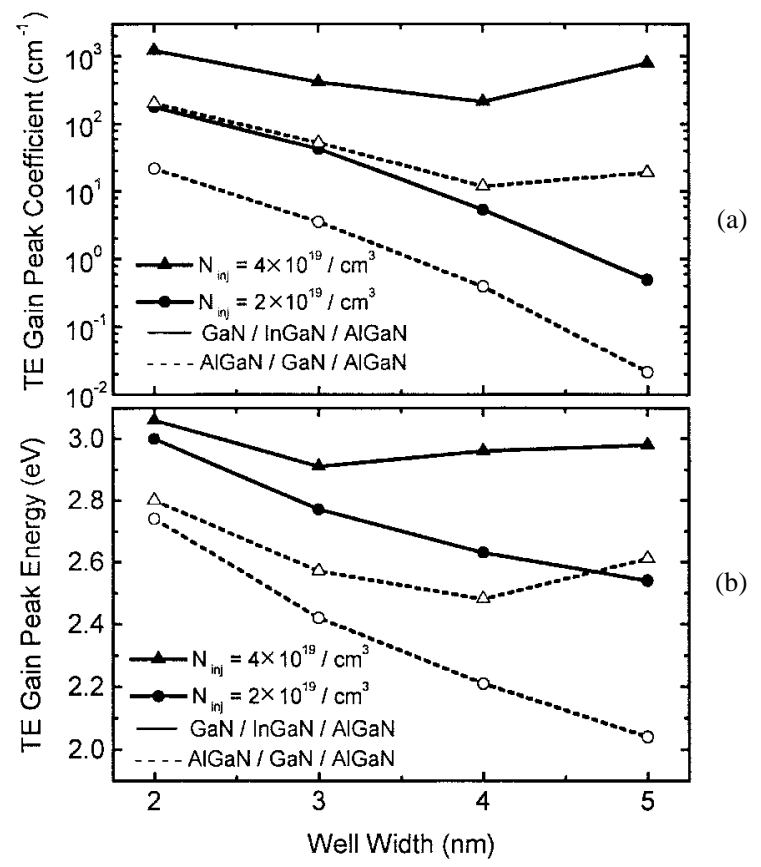

Fig. 9. Well width dependence of the TE-polarized gain (a) peak coefficient and (b) peak energy for the symmetric and asymmetric $\mathrm{In}_{0.2} \mathrm{Ga}_{0.8} \mathrm{~N}$ QW's at $N_{\text {inj }}=2 \times$ and $4 \times 10^{19} \mathrm{~cm}^{-3}$.

well width [39]. The increase of the 5.0-nm InGaN QW peak gain coefficient and emission energy with respect to those of the 4.0-nm QW reflect such an action.

We depict in Fig. 9(b) the calculated TE-polarized peak gain energy for InGaN QW's of both kinds of symmetry. At $N_{\text {inj }}=2 \times 10^{19} \mathrm{~cm}^{-3}$, we note the peak gain energy reveals a spectral blue shift as the well width decreases. This phenomenon clearly reflects the quantization effect. Due to the less efficient carrier confinement in the symmetric InGaN QW, the action of charge screening of the piezoelectric field $\left(E_{z}\right)$ is inferior to that in the asymmetric QW. The action of PQCSE, therefore, results in a spectral red shift of the symmetric InGaN QW optical gain spectrum with respect to that of the asymmetric $\mathrm{QW}$. This red shift is more pronounced at larger well width $\left(L_{w}\right)$ since the second order effect of PQCSE scales as $E_{z}^{2} L_{w}^{4}$ [8]. We finally note a concave well width dependence of peak gain energy at high carrier injection of $N_{\mathrm{inj}}=4 \times 10^{19}$ $\mathrm{cm}^{-3}$. These observations bear the same origin of band-filling and screening as those shown in Fig. 9(a). A spectral blue shift due to the filling of $n=2$ subband is shown to take place at a well width $L_{w}>4 \mathrm{~nm}$.

From a designer's point of view, it is desirable to have a compilation of the optical gain spectra for the targeting active-layer structures. Shown in Fig. 10 are the well width dependence of the optical gain spectra of the $\mathrm{InGaN}_{\mathrm{QW}}$ calculated for both kinds of symmetry. At $N_{\mathrm{inj}}=4 \times 10^{19}$ $\mathrm{cm}^{-3}$, one order of magnitude increase in the optical gain coefficient can be achieved by taking advantage of the stronger confinement potential and charge screening of PQCSE in the asymmetric InGaN QW. This combined effect also results in a spectral blue shift of the asymmetric InGaN QW gain spectra with respect to that of the symmetric QW. We also note that with an increase of the injection carrier density from

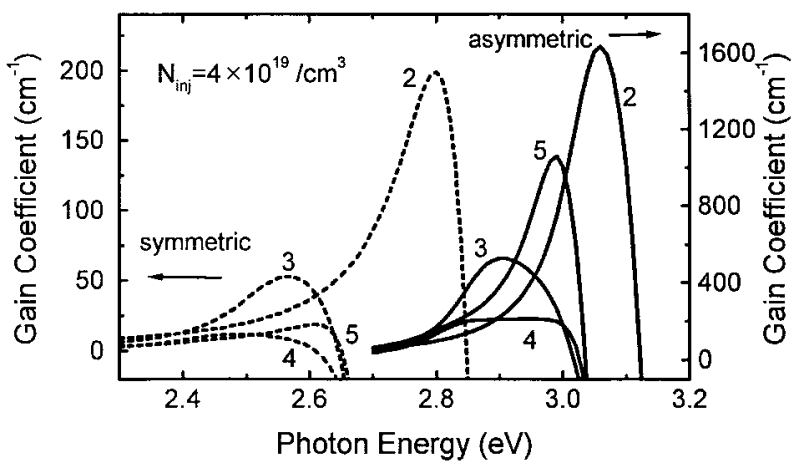

Fig. 10. Well width dependence of the optical gain spectra for the symmetric and asymmetric $\mathrm{In}_{0.2} \mathrm{Ga}_{0.8} \mathrm{~N}$ QW at $N_{\mathrm{inj}}=4 \times 10^{19} \mathrm{~cm}^{-3}$.

$2 \times$ to $4 \times 10^{19} \mathrm{~cm}^{-3}$, a tenfold increase in the optical gain coefficient can be achieved. At the same time, a wide current tunability of gain peak energy on the order of $0.2 \mathrm{eV}$ can also be assured. Such effects are therefore very useful for the design of InGaN QW lasers.

\section{CONCLUSION}

In summary, the EL spectra of strained $\mathrm{GaN}-\mathrm{In}_{0.2} \mathrm{Ga}_{0.8} \mathrm{~N}-\mathrm{Al}_{0.2} \mathrm{Ga}_{0.8} \mathrm{~N}$ QW and $\mathrm{Zn}: \mathrm{Si}_{\mathrm{S}} \mathrm{In}_{0.06} \mathrm{Ga}_{0.94} \mathrm{~N}$ DH emitters have been analyzed using a pulsed current excitation technique. A spectral blue shift of $80 \mathrm{meV}$ in the InGaN QW emitter is observed as the injection current increases from $1 \mathrm{~mA}$ to $1 \mathrm{~A}$. It is shown the emission spectrum of a piezostrained InGaN QW is determined by a competition between a spectral red shifting mechanism of PQCSE and bandgap renormalization, and a blue shifting mechanism of band-filling and Poisson (charge screening) effects. The In compositional inhomogeneity is shown responsible for the inhomogeneous spectral broadening at high carrier injection but bears no relation to the bandgap narrowing effect. The self-consistent analysis indicates the spectral red-shifting of the InGaN QW emission with respect to the bulk bandgap energy of InGaN is due to the dominant PQCSE effect. The spectral blue-shifting of the InGaN QW emission with injection current is ascribed to the combined effects of band-filling and diminishing of PQCSE. It is shown the asymmetric $\mathrm{GaN}-\mathrm{InGaN}-\mathrm{AlGaN}$ QW's can provide superior carrier confinement and screening of the piezoelectric field than does the symmetric InGaN-AlGaN QW's. Using an asymmetric InGaN QW design, one order of magnitude enhancement in the TE-polarized optical gain can be achieved compared with the symmetric InGaN QW case.

\section{APPENDIX}

Following [28], the block diagonalized form of the $6 \times 6$ valence band RSP Hamiltonian matrix can be written as

$$
H_{6 \times 6}=\left[\begin{array}{cc}
H^{U} & 0 \\
0 & H^{L}
\end{array}\right]
$$


where $H^{U}$ and $H^{L}$ are $3 \times 3$ matrices bearing the relation of $H^{U}=\left(H^{L}\right)^{*}$, and

$$
\begin{aligned}
H^{U} & =\left[\begin{array}{ccc}
F & K_{t} & -i H_{t} \\
K_{t} & G & \Delta-i H_{t} \\
i H_{t} & \Delta+i H_{t} & \lambda
\end{array}\right] \\
F & =\Delta_{1}+\Delta_{2}+\lambda+\theta \\
G & =\Delta_{1}-\Delta_{2}+\lambda+\theta \\
\lambda & =\frac{\hbar^{2}}{2 m_{0}}\left(A_{1} k_{z}^{2}+A_{2} k_{t}^{2}\right)+\lambda_{\varepsilon} \\
\lambda_{\varepsilon} & =D_{1} \varepsilon_{z z}+D_{2}\left(\varepsilon_{x x}+\varepsilon_{y y}\right) \\
\theta & =\frac{\hbar^{2}}{2 m_{0}}\left(A_{3} k_{z}^{2}+A_{4} k_{t}^{2}\right)+\theta_{\varepsilon} \\
\theta_{\varepsilon} & =D_{3} \varepsilon_{z z}+D_{4}\left(\varepsilon_{x x}+\varepsilon_{y y}\right) \\
K_{t} & =\frac{\hbar^{2}}{2 m_{0}} A_{5} k_{t}^{2} \\
H_{t} & =\frac{\hbar^{2}}{2 m_{0}} A_{6} k_{z} k_{t} \\
\Delta & =\sqrt{2} \Delta_{3} .
\end{aligned}
$$

We note $k_{t}=\sqrt{k_{x}^{2}+k_{y}^{2}}$ is the magnitude of the wavevector in the $k_{x}-k_{y}$ plane.

The piezoelectric field in a strained $\mathrm{InGaN}$ QW is given by [41]

$$
E_{z}=-\frac{2 d_{31}}{\varepsilon_{0} \varepsilon_{r}}\left(C_{11}+C_{12}-\frac{2 C_{13}^{2}}{C_{33}}\right) \varepsilon_{x x}
$$

The strain element is defined as

$$
\begin{aligned}
\varepsilon_{x x} & =\varepsilon_{y y}=\frac{a_{0}-a}{a} \\
\varepsilon_{z z} & =-\frac{2 C_{13}}{C_{33}} \varepsilon_{x x}
\end{aligned}
$$

where $a_{0}$ and $a$ are the lattice constants of the substrate and the well material, respectively.

The TE-polarized matrix element is given as follows:

$$
\left|\left(M_{x}\right)_{n m}^{\sigma}\left(k_{t}\right)\right|^{2}=\frac{\left|\left\langle S\left|p_{x}\right| X\right\rangle\right|^{2}}{4}\left\{\left\langle\phi_{n} \mid g_{m}^{(1)}\right\rangle^{2}+\left\langle\phi_{n} \mid g_{m}^{(2)}\right\rangle^{2}\right\}
$$

for $\sigma=U$, and

$$
\left|\left(M_{x}\right)_{n m}^{\sigma}\left(k_{t}\right)\right|^{2}=\frac{\left|\left\langle S\left|p_{x}\right| X\right\rangle\right|^{2}}{4}\left\{\left\langle\phi_{n} \mid g_{m}^{(4)}\right\rangle^{2}+\left\langle\phi_{n} \mid g_{m}^{(5)}\right\rangle^{2}\right\}
$$

for $\sigma=L$, where $\phi_{n}$ and $g_{m}$ are the conduction and valence subband envelope function, respectively.

The spontaneous emission spectrum is calculated according to $\hbar \omega \gamma_{s p}^{e}(\hbar \omega)$, where

$$
\begin{aligned}
\gamma_{s p}^{e}(\hbar \omega)= & \frac{n_{r}^{2} \omega^{2}}{\pi^{2} \hbar c^{2}} g_{s p}^{e}(\hbar \omega) \\
g_{s p}^{e}(\hbar \omega)= & \frac{2 q^{2}}{n_{r} c \varepsilon_{0} m_{0}^{2} \omega L_{w}} \sum_{\sigma=U, L} \sum_{n, m} \int \frac{k_{t} d k_{t}}{2 \pi} \\
& \times\left|\left(M_{e}\right)_{n m}^{\sigma}\left(k_{t}\right)\right|^{2} \times f_{n}^{c}\left(k_{t}\right) \times\left[1-f_{\sigma, m}^{v}\left(k_{t}\right)\right] \\
& \times \frac{\hbar \gamma / \pi}{\left[E_{\sigma, n m}^{c v}\left(k_{t}\right)-\hbar \omega\right]^{2}+(\hbar \omega)^{2}}
\end{aligned}
$$

and $e$ is the polarization direction.
The optical gain spectrum is calculated according to

$$
g^{e}(\hbar \omega)=g_{s p}^{e}(\hbar \omega)\left[1-\exp \left(\frac{\hbar \omega-\Delta F}{k_{B} T}\right)\right]
$$

where $\Delta F$ measures the quasi Fermi-level difference in the conduction and valence band, respectively, and $f$ 's in (A8) are the corresponding Fermi-Dirac distribution.

We approximate the effects of In inhomogeneity on the optical spectrum $G$ by a statistical average of a normal distribution [14], where

$$
\begin{aligned}
& G_{i n h}\left(\omega, \varepsilon, \gamma, N_{\mathrm{inj}}, T\right) \\
& \quad=\int d x P(x) \times G\left(\omega, \varepsilon, \gamma, N_{\mathrm{inj}}, T\right) .
\end{aligned}
$$

$P(x)=(\sqrt{2 \pi \sigma})^{-1} \exp \left\{-\left[\left(x-x_{0}\right) /(\sqrt{2 \sigma})\right]^{2}\right\}$ is the normal distribution representing the In compositional variation. The distribution is characterized by a mean value of $x_{0}$ and a standard deviation of $\sigma$.

\section{ACKNOWLEDGMENT}

The authors wish to thank Dr. L.-H. Lou at Nichia-Unilux Corp., Taiwan, R.O.C., for sample support, and Prof. I. Akasaki at Mejio University, Japan, for discussion.

\section{REFERENCES}

[1] S. Nakamura, "InGaN multiquantum-well-structure laser diodes with GaN-AlGaN modulation-doped strained-layer superlattice," IEEE J. Select. Topics Quantum Electron., vol. 4, pp. 483-489, 1998.

[2] J. Han, M. H. Crawford, R. J. Shul, J. J. Figiel, M. Banas, L. Zhang, Y. K. Song, H. Zhou, and A. V. Nurmikko, "AlGaN/GaN quantum well ultraviolet light emitting diodes," Appl. Phys. Lett., vol. 73, pp. 1688-1690, 1998.

[3] T. Mukai, H. Narimatsu, and S. Nakamura, "Amber InGaN-based lightemitting diodes operated at high ambient temperature," Jpn. J. Appl. Phys., vol. 37, pp. L479-L481, 1998.

[4] S. Nakamura, "InGaN-based blue laser diodes," IEEE J. Select. Topics Quantum Electron., vol. 3, pp. 712-718, 1997.

[5] S. Nakamura, M. Senoh, S. I. Nagahama, N. Iwasa, T. Yamada, T. Matsushita, H. Kiyoku, Y. Sugimoto, T. Kozaki, H. Umemoto, M. Sano, and K. Chocho, "InGaN-GaN-AlGaN-based laser diodes with modulation-doped strained-layer superlattices," Jpn. J. Appl. Phys., vol. 36, pp. L1568-L1571, 1997.

[6] — "InGaN-GaN-AlGaN-based laser diodes with cleaved facets grown on GaN substrates," Appl. Phys. Lett., vol. 73, pp. 832-834, 1998.

[7] W. W. Chow, S. W. Koch, and M. Sargent, III, Semiconductor Laser Physics. Berlin, Germany: Springer, 1994.

[8] D. L. Smith and C. Mailhiot, "Piezoelectric effects in strained-layer superlattices," J. Appl. Phys., vol. 63, pp. 2717-2719, 1988.

[9] Y. M. Sirenko, J. B. Jeon, B. C. Lee, K. W. Kim, M. A. Littlejohn, M. A. Stroscio, and G. J. Iafrate, "Hole scattering and optical transitions in wide-band-gap nitrides: Wurtzite and zinc-blend structures," Phys. Rev. B, vol. 55, pp. 4360-4375, 1997.

[10] Y. C. Yeo, T. C. Chong, M.-F. Li, and W. J. Fan, "Electronic band structures and optical gain spectra of strained wurtzite $\mathrm{GaN} / \mathrm{Al}_{x} \mathrm{Ga}_{1-x} \mathrm{~N}$ quantum-well lasers," IEEE J. Quantum Electron., vol. 34, pp. 526-534, 1998.

[11] S. Nakamura, M. Senoh, N. Iwasa, and S.-I. Nagahama, "High-power InGaN single-quantum-well-structure blue and violet light-emitting diodes," Appl. Phys. Lett., vol. 67, pp. 1868-1870, 1995.

[12] _ "High-brightness InGaN blue, green, and yellow light-emitting diodes with quantum well structures," Jpn. J. Appl. Phys., vol. 34, pp. L797-L799, 1995.

[13] S. Chichibu, T. Sota, K. Wada, and S. Nakamura, "Exciton localization in InGaN quantum wells," J. Vac. Sci. Technol. B, vol. 16, no. 4, pp. 2204-2214, 1998.

[14] W. W. Chow, A. F. Wright, A. Girndt, F. Jahnke, and S. W. Koch, "Microscopic theory of gain for an InGaN/GaN quantum well laser," Appl. Phys. Lett., vol. 71, pp. 2608-2610, 1997. 
[15] S. F. Chichibu, H. Marchand, M. S. Minsky, S. Keller, P. T. Fini, J. P. Ibbetson, S. B. Fleischer, J. S. Speck, J. E. Bowers, E. Hu, U. K. Mishra, S. P. DenBaars, T. Deguchi, T. Sota, and S. Nakamura, "Emission mechanism of bulk and InGaN quantum wells prepared by lateral epitaxial overgrowth," Appl. Phys. Lett., vol. 74, pp. 1460-1462, 1999.

[16] C. Wetzel, T. Takeuchi, H. Amano, and I. Akasaki, "Piezoelectric Franz-Keldysh effect in strained GaInN/GaN heterostructures," J. Appl. Phys., vol. 85, pp. 3786-3791, 1999.

[17] H. Kollmer, J. S. Im, S. Heppel, J. Off, F. Scholz, and A. Hangleiter, "Intra- and inter-well transitions in GaInN/GaN multiple quantum wells with built-in piezoelectric fields," Appl. Phys. Lett., vol. 74, pp. 82-84, 1999.

[18] A. Bykhovski, B. Gelmont, and M. Shur, "The influence of the straininduced electric field on the charge distribution in GaN-AlN-GaN structure," J. Appl. Phys., vol. 74, pp. 6734-6739, 1993.

[19] T. Takeuchi, C. Wetzel, S. Yamaguchi, H. Sakai, H. Amano, I. Akasaki, Y. Kaneko, S. Nakagawa, Y. Yamaoka, and N. Yamada, "Determination of piezoelectric fields in strained GaInN quantum well using the quantum-confined Stark effect," Appl. Phys. Lett., vol. 73, pp. 1691-1693, 1998

[20] S. F. Chichibu, A. C. Abare, M. S. Minsky, S. Keller, S. B. Fleischer, J. E. Bowers, E. Hu, U. K. Mishra, L. A. Coldren, S. P. DenBarrs, and T. Sota, "Effective band gap inhomogeneity and piezoelectric field in InGaN/GaN multiquantum well structures," Appl. Phys. Lett., vol. 73, pp. 2006-2008, 1998.

[21] H. S. Kim, J. Y. Lin, H. X. Jiang, W. W. Chow, A. Botchkarev, and H. Morkoç, "Piezoelectric effects on the optical properties of GaN/AlGaN multiple quantum wells," Appl. Phys. Lett., vol. 73, pp. 3426-3428, 1998.

[22] J. S. Im, H. Kollmer, J. Off, A. Sohmer, F. Scholz, and A. Hangleiter, "Reduction of oscillator strength due to piezoelectric fields in GaN/AlGaN quantum wells, " Phys. Rev. B, vol. 57, pp. R9435-R9438, 1998.

[23] E. T. Yu, X. Z. Dang, L. S. Yu, D. Qiao, P. M. Asbeck, S. S. Lau, G. J. Sullivan, and J. M. Redwing, "Schottky barrier engineering in III-V nitrides via the piezoelectric effects," Appl. Phys. Lett., vol. 73, pp. 1880-1882, 1998.

[24] R. Gaska, J. W. Yang, A. Osinsky, A. D. Bykhovski, and M. S. Shur, "Piezoeffect and gate current in AlGaN/GaN high electron mobility transistors," Appl. Phys. Lett., vol. 71, pp. 3673-3675, 1997.

[25] M. B. Nardelli, K. Rapcewicz, and J. Bernholc, "Polarization field effects on the electron-hole recombination dynamics in $\mathrm{In}_{0.2} \mathrm{Ga}_{0.8} \mathrm{~N} / \mathrm{In}_{1-x} \mathrm{Ga}_{x} \mathrm{~N}$ multiple quantum wells," Appl. Phys. Lett., vol. 71, pp. 3135-3137, 1997.

[26] S.-H. Park and D. Ahn, "Many-body effects on optical gain in strained hexagonal and cubic GaN/AlGaN quantum well lasers," Appl. Phys. Lett., vol. 71, pp. 398-401, 1997

[27] S.-H. Park and S.-L. Chuang, "Many-body optical gain of wurtzite GaNbased quantum well lasers and comparison with experiment," Appl. Phys. Lett., vol. 72, pp. 287-289, 1998.

[28] S. L. Chuang, "Optical gain of strained wurtzite GaN quantum well lasers," IEEE J. Quantum Electron., vol. 32, pp. 1791-1800, 1996.

[29] J. Wang, J. B. Jeon, Y. M. Sirenko, and K. W. Kim, "Piezoelectric effect on optical properties of pseudomorphically strained wurtzite $\mathrm{GaN}$ quantum wells," IEEE Photon. Technol. Lett., vol. 9, pp. 728-730, 1997.

[30] S. Chichibu, T. Azuhata, T. Sota, and S. Nakamura, "Spontaneous emission of localized excitons in InGaN single and multiquantum well structures," Appl. Phys. Lett., vol. 69, pp. 4188-4190, 1996.

[31] S. Nakamura, "GaN-based blue/green semiconductor laser," IEEE Select. Topics Quantum Electron., vol. 3, pp. 435-442, 1997.

[32] S. Nakamura, T. Mukai, and M. Senoh, "Candela-class high-brightness InGaN/AlGaN double-heterstructure blue-light-emitting diodes," Appl. Phys. Lett., vol. 64, pp. 1687-1689, 1994.

[33] L.-H. Peng, C.-W. Chuang, and L.-H. Lou, "Piezoelectric effects in the optical properties of strained InGaN quantum wells," Appl. Phys. Lett., vol. 74, pp. 795-797, 1999.

[34] P. Perlin, M. Osinski, P. G. Eliseev, V. A. Smagley, J. Mu, M Banas, and P. Sartori, "Low-temperature study of current and electroluminescence in InGaN/AlGaN/GaN double-heterostructure blue lightemitting diode," Appl. Phys. Lett., vol. 69, pp. 1680-1682, 1996.
[35] J. P. Basrur, F. S. Choa, P.-L. Liu, J. Sipior, G. Rao, G. M. Carter, and Y. J. Chen, "The process and efficiency of ultraviolet generation from gallium nitride blue light emitting diodes," Appl. Phys. Lett., vol. 71, pp. 1385-1387, 1997.

[36] H. C. Casey, Jr., J. Muth, S. Krishnankutty, and J. M. Zavada, "Dominance tunneling current and band filling in InGaN/AlGaN double hetero-structure blue light-emitting diodes," Appl. Phys. Lett., vol. 68, pp. 2867-2869, 1996.

[37] M. Smith, J. Y. Lin, H. X. Jiang, and M. A. Khan, "Room temperature intrinsic optical transition in GaN epilayers: the band-to-band versus excitonic transitions," Appl. Phys. Lett., vol. 71, pp. 635-637, 1997.

[38] G. Y. Zhao, G. Yu, T. Egawa, J. Watanabe, T. Jimbo, and M. Umeno, "Energy-gap narrowing in a current injected InGaN/AlGaN surface light emitting diode," Appl. Phys. Lett., vol. 71, pp. 2424-2426, 1997.

[39] A. Tomita and A. Suzuki, "Carrier-induced lasing wavelength shift for quantum well laser diodes," IEEE J. Quantum Electron., vol. 23, pp. 1155-1159, 1987.

[40] G. Trankle, H. Leier, A. Forchel, H. Haug, C. Ell, and G. Weimann, "Dimensionality dependence of the band-gap renormalization in twoand three-dimensional electron-hole plasma," Phys. Rev. Lett., vol. 58, pp. 419-422, 1987.

[41] T. Takeuchi, S. Sota, M. Katsuragawa, M. Komori, H. Takeuchi, H. Amano, and I. Akaksaki, "Quantum-confined Stark effect due to piezoelectric fields in GaInN strained quantum wells," Jpn. J. Appl. Phys., vol. 36, pp. L382-L385, 1997.

[42] Properties of Group III Nitrides, J. H. Edgar, Ed. London, U.K. INSPEC/IEE, 1994.

[43] S. Nakamura and G. Fasol, The Blue Laser Diode. Berlin, Germany: Springer-Verlag, 1997

[44] Y. C. Yeo, T. C. Chong, and M. F. Li, "Electronic band structures and effective-mass parameters of wurtzite GaN and InN," J. Appl. Phys. vol. 83, pp. 1429-1436, 1998

[45] S. L. Chuang and C. S. Chang, "A band-structure model of strained quantum-well wurtzite semiconductor," Semiconduct. Sci. Technol., vol. 12, pp. 252-263, 1997.

[46] F. D. Sala, A. D. Carlo, P. Lugli, F. Bernardini, V. Fiorentini, R. Scholz, and J.-M. Jancu, "Free-carrier screening of polarization fields in wurtzite GaN/InGaN laser structures," Appl. Phys. Lett., vol. 74, pp. 2002-2004, 1999.

[47] K. P. O'Donnel, T. Breitkopf, H. Kalt, W. Van der Stricht, I. Moerman, P. Demeester, and P. G. Middleton, "Optical linewidths of InGaN light emitting diodes and epilayers," Appl. Phys. Lett., vol. 70, pp. 1843-1845, 1997.

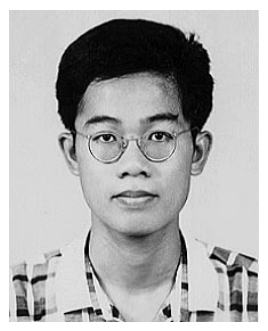

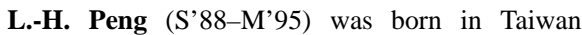
R.O.C., in 1964. He received the bachelor's degree in electrical engineering from National Taiwan University in 1986, and the Master's and Ph.D. degree in applied physics from Harvard University, Cambridge, MA, in 1989 and 1994, respectively.

$\mathrm{He}$ was a Visiting Scientist at Massachusetts Institute of Technology, Cambridge, in 1994 and Post-Doctoral Research Fellow at Oak Ridge National Laboratory, TN, in 1995. Since 1995, he has been with the Graduate Institute of ElectroOptical Engineering and Department of Electrical Engineering in National Taiwan University and is currently an Associate Professor. His research interest includes semiconductor optics and nonlinear optics.

Dr. Peng is a member of the American Physics Society.

Y.-C. Hsu, photograph and biography not available at the time of publication.

C.-W. Chuang, photograph and biography not available at the time of publication. 